\title{
Sistemas de acceso a la información telemática
}

\author{
José Antonio Merlo Vega \\ Universidad de Salamanca
}

\subsection{Resumen}

Internet ofrece distintos sistemas para acceder a la información. Se exponen los diferentes tipos de documentos que pueden ser localizados en Internet, así como las distintas fuentes de información de Internet. Se ofrece una clasificación de los sistemas de acceso a la información telemática a partir del tratamiento y el análisis que recibe la información, dividiéndose estos sistemas en tratamiento intelectual y automático y sistemas con o sin análisis documental. Se estudian los distintos aspectos que intervienen en el proceso de búsqueda de información en Internet, estructurados bajo las categorías qué buscar, dónde buscar y cómo buscar.

Palabras clave: Internet. Sistemas de búsqueda. Información digital. Buscadores. Índices.

\subsection{Abstract}

Internet provides with different systems for information access. The various types of documents and sources of information that can be found in Internet are examined. A classification of the telematic access information systems is offered, based on the treatment and analysis that information receives. The systems are divided according to two criteria. First, if they received an intellectual or automatic treatment; second, if they have been properly catalogued or not. Finally, the different aspects of the searching process in the Internet are studied and classified into three categories: what, where and how to search.

Keywords: Internet. Searching. Browsing. Digital information. Search engines. Indexes.

\section{Información electrónica e información telemática}

En un sentido amplio se puede denominar información electrónica a aquella que está registrada sobre un soporte para cuya consulta son necesarios dispositi- 
vos electrónicos. Desde un punto de vista más estricto, se utiliza esta denominación para referirse a los datos registrados sobre soportes ópticos y magnéticos y para las informaciones que están accesibles de manera telemática.

Según los soportes y los sistemas de acceso a la información en ellos registrada será necesario emplear unos dispositivos u otros: ordenadores, lectores de discos ópticos, módems, softwares específicos, etc.

Dada la amplitud semántica del concepto es posible encontrarse con una gran cantidad de sinónimos y acepciones, por lo que es conveniente limitar en la medida de lo posible los términos que definan a cada una de las tipologías existentes. De este modo, el sentido de información electrónica puede y debe especificarse empleando como calificador los diferentes tipos de soporte en los que quedan registradas y/o el medio de acceso a los mismos. Así, habría que hablar de (1):

- Información magnética: la que emplea disquetes o cualquier otro tipo de dispositivos magnéticos como medio de almacenamiento.

- Información óptica: la que utiliza discos compactos como sistema de registro de la información.

- Información telemática: aquella registrada digitalmente a la que se accede a través de una red de telecomunicaciones.

Existe una gran variedad de servicios de información telemática, tales como las bases de datos en línea tradicionales o bases de datos ASCII, el vídeotexto, el teletexto, el audiotexto e Internet. Esta última forma de acceso a la información es en la actualidad el servicio telemático de información más empleado, dadas las facilidades existentes para conectarse a esta red y emplearla como medio para el envío y la recepción de datos.

El acceso a la información a través de Internet es posible gracias a tres aspectos: los protocolos TCP/IP, las direcciones IP y el modelo cliente-servidor. Los protocolos TCP/IP permiten que los ordenadores conectados a Internet puedan entenderse entre sí y, por tanto, enviar y recibir información mutuamente. La direcciones IP posibilitan que los equipos estén identificados, para saber a dónde enviar y de dónde recibir la información. Por último, el modelo cliente-servidor implica que se pueda utilizar la información contenida en determinados ordenadores (servidores) empleando programas o sistemas específicos (clientes) alojados en nuestros ordenadores.

El éxito de Internet reside también en los diferentes formatos de información que permite albergar. Es difícil realizar una única clasificación de los tipos de documentos disponibles en Internet, aunque sí es posible agrupar los tipos de información accesibles en Internet a partir de determinados criterios: 
- Según el sistema de acceso: telnet, hipertexto, ftp, correo electrónico, noticias, BBS, X500, etc.

- Según el formato: ASCII, hipertexto, texto, imágenes, vídeo, sonido, software, etc. (2).

- Según el valor de la información: primaria (información final o válida por sí misma), secundaria (remite a páginas con información primaria: directorios, índices, etc.), terciaria (envía a páginas secundarias: directorios de directorios, etc.).

\section{Acceso a la información telemática}

Como se ha expuesto en los últimos párrafos, en Internet pueden encontrarse documentos de formatos muy distintos. Cada tipo de información tiene varios lugares desde los que ser localizado, es decir, de servicios de información a los que interrogar para acceder a esos documentos. Tomando las divisiones anteriores podría establecerse una clasificación de los servicios de información en línea a partir del tipo de información que ofrecen y, sobre todo, del sistema que se emplea para acceder a la misma. Una manera de sistematizar estos servicios es la siguiente:

- Información accesible a través de telnet: La conexión remota es el único medio de consulta de determinadas informaciones, como ocurre con muchas bases de datos y catálogos bibliotecarios. Sistemas de información como Hytelnet (3) ayudan a localizar este tipo de información, además de ofrecer datos acerca de cómo deben ser consultados los servidores telnet. Por lo general, la información se obtiene después de la consulta de varias páginas organizadas mediante un sistema de menús.

- Información accesible a través de FTP: Internet ofrece acceso a ficheros y programas para transferir vía FTP. Muchos sistemas de búsqueda mantienen una base de datos de ficheros, indicando características de los mismos: sistema operativo, gratuidad, etc. Estos sistemas poseen un motor de búsqueda desde el que realizar las consultas. Estos servicios no sólo localizan los programas o documentos, sino que también permiten su descarga (4).

- Información accesible a través de gopher: Cada vez existen menos servidores con información accesible mediante gopher; no obstante, también hay en la red sistemas para localizar los documentos de este tipo. Estos directorios son útiles porque ayudan a localizar información textual que puede ser útil como fuente de información o como información final. Esto es así sobre todo en instituciones con presencia antigua en la red (5).

- Direcciones de correo electrónico: El correo electrónico es uno de los servicios más empleados de Internet. Es un buen medio para acceder a fuen- 
tes de información como las listas de distribución o para obtener informaciones directamente. En la red se encuentran servicios especializados en ofrecer los correos electrónicos gracias a sus bases de datos y motores de búsqueda. La utilidad de cada uno de estos sistemas dependerá del número de usuarios recogidos en su base de datos (6).

- Páginas amarillas: Se denominan así a los servicios que ofrecen información de empresas. Los datos que se obtienen pueden ser simplemente dirección, teléfono, etc., o bien su correo electrónico e información sobre las mismas, dependiendo del servicio concreto que se esté consultando. Por lo general, son muy parecidas a las guías telefónicas impresas, incluyendo los mismos datos que estas fuentes impresas (7).

- Páginas blancas: Presentan muchas similitudes con las anteriores, con la diferencia de que en este caso se ofrece información sobre personas. Cualquier servicio de información que permita localizar personas desde una base de datos puede llamarse Páginas blancas. Como ocurría en el tipo anterior, dependiendo del servicio que se consulte se localizará un tipo de información u otra. Por lo general, actúan a modo de "Quién es quién", como directorios de personas (8).

- Informaciones enviadas a grupos de noticias: Los newsgroups son a menudo una fuente de información útil. Asimismo, es posible consultar servicios de información que han recogido mensajes enviados a estos grupos para cargarlos en una base de datos accesible sin restricciones. En los miles de grupos existentes la temática de los mensajes noticias es amplia, predominando los aspectos de ocio y los informales (9).

- Informaciones enviadas a las listas de distribución: Las listas de distribución reciben informaciones de sumo interés. Estas listas suelen tratar de temas especializados, por lo que la consulta a sus archivos de mensajes puede ser un buen medio para localizar informaciones. Dentro de los servicios de consulta de este tipo de información es posible encontrarse con sistemas que localizan mensajes enviados a una única lista o bien bases de datos de mensajes de varias listas (10).

- Imágenes, gráficos y mapas: La información gráfica también está presente en Internet y, por consiguiente, también existen sistemas para localizar directamente esta información, ya se trate de fotografías, mapas, animaciones o imágenes de cualquier tipo. Las imágenes contenidas en estos sistemas son de dominio público, por lo que pueden ser descargadas por los usuarios (11).

- Archivos de audio: Como ocurre con la imagen, el sonido también está presente en Internet: en distintos formatos, para acompañar páginas, para 
transmitir informaciones o para ser descargados. Los archivos de audio se emplean de varias formas y en varios soportes. Por ello, son muy útiles los servicios que recogen este tipo de información. Entre sus aplicaciones está la retransmisión en directo de acontecimientos y programas de radio (12).

- Canales IRC: Los chat o conversaciones en directo a través de Internet, ya sea mediante texto o voz, también pueden ser considerados una fuente de información, ya que existen muchos canales IRC en los cuáles se intercambian datos e informaciones de interés. Internet dispone de servicios de información desde los cuáles localizar estos canales y poder participar en ellos para solicitar información (13).

- Archivos de vídeo y web cams: Al igual que el resto de los formatos el vídeo también está accesible en Internet, ya sea únicamente imagen en movimiento o también con sonido. Además de los archivos de vídeo, se puede examinar televisión en directo, retransmisiones de acontecimientos y cámaras que ofrecen lo que captan a través de la red (webcams). La utilidad de este procedimiento como fuente de información dependerá del servicio consultado (14).

- Documentos hipertexto: Un altísimo porcentaje de la información depositada en Internet está en formato HTML, es decir, como páginas web. La calidad de los documentos así editados y las facilidades del hipertexto para desplazarse de un documento a otro han hecho de los documentos web la principal fuente de información. Los dispositivos de localización de páginas web son múltiples y van desde los buscadores o bases de datos de páginas web, hasta los índices de recursos organizados por temas, como se expondrá más adelante.

\section{Servicios de información en línea y tratamiento documental}

Por lo general, los servicios de acceso a la información telemática se estudian desde un punto de vista informático, o bien desde la forma en que estructuran su contenido. No obstante, es posible establecer una clasificación de los sistemas de información accesibles por procedimientos telemáticos atendiendo al tipo de tratamiento documental que la información recibe en los distintos sistemas. A partir de este criterio es posible separar los sistemas de información entre los que realizan un tratamiento intelectual, es decir, humano, y aquellos que reciben un tratamiento automático. Además, cabe otra clasificación dentro de la anterior, en esta ocasión partiendo de si se realiza o no análisis documental de la información.

Atendiendo a estos dos criterios — tratamiento y análisis documental— los servicios de información telemática se podrían dividir entre los servicios que realizan un tratamiento de la información intelectual y los que lo realizan de forma

Scire. 4 : 2 (jul.-dic. 1998) 79-103. 
automática. A su vez, ambos se subdividen entre los que efectúan algún tipo de análisis documental y los que no aplican ningún tipo de análisis documental. Los tipos de servicios de información telemática resultantes a partir de esta sistematización se sintetizan en la tabla I:

\begin{tabular}{|l|l|l|}
\cline { 2 - 3 } \multicolumn{1}{c|}{} & Con análisis documental & Sin análisis documental \\
\hline Tratamiento & Bases de datos & Índices temáticos \\
& Catálogos de bibliotecas & Índices geográficos \\
& Bibliografías & Recopilaciones \\
& Directorios analíticos & Anillos temáticos \\
& Bibliotecas digitales & Portales \\
& & Sitios web \\
\hline $\begin{array}{l}\text { Tratamiento } \\
\text { automático }\end{array}$ & & Buscadores \\
\hline
\end{tabular}

Tabla I. Clasificación de los servicios de información telemática

En muchas ocasiones estos servicios de información son híbridos, ya que en un mismo lugar se reúnen simultáneamente fuentes de información distintas: índices y buscadores, portales y selecciones, etc. En otros casos el problema es que un servicio de información pasa por una fase automática y por otra intelectual, bien porque los documentos son recopilados por programas automáticos y luego tratados por personas, bien porque las informaciones son elaboradas por medios humanos y posteriormente descargadas en sistemas automáticos de recuperación.

A pesar de la existencia de los sistemas mixtos, es preferible describir cada uno de los servicios de información telemática por separado, a fin de conseguir más claridad en la articulación de los métodos de los que dispone un usuario de Internet para acceder a la información depositada en la red.

Para una mejor comprensión de las categorías expuestas se ofrecen ejemplos de cada una de ellas, con la intención de que se puedan comprobar las características de las distintas fuentes de información comentadas. 


\subsection{Servicios de información con tratamiento intelectual}

Este tipo de servicios o de fuentes de información telemática son elaboradas de manera íntegra por personas, quienes deciden a qué categoría pertenece un documento o bien que relación tiene una determinada página web con otra. La utilidad de la actividad intelectual en la elaboración de productos bibliográficos telemáticos oscilará entre los productos más exhaustivos en los que existe un análisis detallado de las informaciones hasta los más simples en los cuáles solamente se faciliten relaciones de documentos.

\subsubsection{Tratamiento intelectual con análisis documental}

Se clasifican así a los sistemas que ofrecen una información para cuya elaboración se han seguido técnicas de análisis documental (indización, catalogación, etc.) y se han empleado lenguajes documentales (tesauros, clasificaciones, etc.). Son fuentes de información tradicionales, en cuanto a que no son un producto aparecido con Internet, pero cuyo acceso se realiza por procedimientos telemáticos, de ahí que se incluyan dentro del conjunto de sistemas de información presentes en Internet.

Este tipo de servicios de información han sido desarrollados mediante un trabajo humano, por tanto intelectual, en el cual se han aplicado las técnicas de descripción documental, tanto formal como de contenido. Por este motivo, es necesario separarlas del resto de los recursos de información que se encuentran en Internet.

Las fuentes de información en las cuáles existe tratamiento intelectual y análisis documental son las siguientes:

\subsubsection{Bases de datos}

Desde Internet se puede acceder a un gran número de bases de datos de temática multidisciplinar. Dependiendo de la información y de la entidad productora el acceso será libre o restringido, y gratuito o mediante pago. El valor de estos sistemas como fuente de información es incalculable, tanto por la facilidad en el acceso a bases de todo el mundo, como por la variedad de informaciones en ellas contenidas. Véanse por ejemplo:

- Internet Movie Database: http://spanish.imdb.com

- COMPLUDOC (BUCM): http://www.ucm.es/BUCM/complu/

- TEMPUS (INE): http://www.ine.es/tempus/cgi-bin/iti 


\subsubsection{Catálogos de bibliotecas}

Los catálogos de bibliotecas se emplean como una fuente de información bibliográfica y como un medio de acceso al documento primario. Los catálogos de las grandes bibliotecas son una buena vía para obtener datos sobre obras, autores, materias, etc. Con la simplificación de los sistemas de consulta a través de web, estas fuentes de información han aumentado en un alto porcentaje su uso y su valor como medio de información. Véanse por ejemplo:

- Biblioteca Nacional: http://www.bne.es/esp/cat.htm

- Bibliotecas Públicas del Estado: http://www.mcu.es/bpe/bpe.html

- Catàleg Col-lectiu de les Universitats de Catalunya: http://www.cbuc.es/ccuc/

\subsubsection{Bibliografías}

En muchas ocasiones las fuentes de información telemática no difieren apenas de las impresas, como ocurre con las bibliografías. Este servicio de información consiste en la recopilación de referencias de documentos de cualquier tipo y en cualquier formato. Las bibliografías electrónicas, como las impresas, facilitan la referencia de documentos, con la evidente diferencia, de que, gracias a la tecnología hipertexto, las bibliografías disponibles en Internet pueden enlazar con el documento al que están representando. Véanse por ejemplo:

- Library \& Information Science: General Resources: http://www.ifla.org/II/libdoc.htm

- Bibliografía sobre bibliobuses (BCyL): http://www.bcl.jcyl.es/cgibin/abweb/L1/T5

- El Faro: artículos y documentos en línea: http://nutabe.udea.edu.co/ elfaro/arti.html

\subsubsection{Directorios analíticos}

Esta importante fuente de información, también llamadas guías temáticas, recopilan y comentan recursos de especial interés o calidad. Organizan la información por grandes temas, los cuáles se van subdividiendo paulatinamente, hasta llegar a una lista específica sobre la materia elegida. En la relación última suelen aparecer comentarios de recursos seleccionados por tratarse de directorios de enlaces o páginas de utilidad por la información que ofrecen. Suelen incorporar también un sistema de búsqueda de forma directa. En estos sistemas el análisis documental aparece en la serie de descriptores que se ofrecen y en el resumen indicativo que se realiza de los recursos seleccionados. Véanse por ejemplo:

- The Argus Clearinghouse: http://www.clearinghouse.net 
- BUBL Link: http://bubl.ac.uk/link

- DARWIN: http://www.kronosdoc.com/darwin/

\subsubsection{Bibliotecas digitales}

Se trata de colecciones de documentos en formato digital, organizadas conforme a criterios documentales y dispuestas para su consulta por los usuarios a través de Internet o de una red conectada a Internet. Son, por tanto, fuentes de información en línea, realizadas por personas con conocimientos biblioteconómicos, los cuáles son aplicados para la descripción y organización de los documentos. El grado de normalización del análisis efectuado en estas bibliotecas dependerá de sus responsables, aunque en la actualidad se tiende a la uniformidad en los soportes y los procedimientos (15). Véanse por ejemplo:

- Bibliotheca Universalis: http://www.culture.fr/g7/eng/aceuil2.htm

- Biblioteca Digital de Catalunya: http://www.cbuc.es/bdc/

- California Digital Library: http://www.cdlib.org

\subsubsection{Tratamiento intelectual sin análisis documental}

A causa del crecimiento imparable de documentos disponibles en Internet se hizo necesario organizar las páginas web para poder acceder a ellas a partir de criterios específicos, como el tema de los documentos o el área geográfica de procedencia. Esta actividad se realiza de forma manual, es decir intelectual, ya que en el proceso de organización de las páginas no intervienen máquinas, sino personas, que deciden a qué categoría asignar cada página.

Los documentos contenidos en las fuentes de información de este apartado no reciben análisis documental en el sentido estricto. A lo sumo se le asigna una clasificación o categoría, pero no se utiliza un lenguaje controlado ni se realizan descripciones del contenido o la forma de los documentos web.

Las fuentes de información en las que existe tratamiento intelectual pero no análisis documental son las siguientes:

\subsubsection{1. Índices temáticos}

Bases de datos de documentos disponibles en Internet organizados de forma temática, partiendo de jerarquías genéricas para ir especificándose las materias página a página. Ofrecen listados de recursos web y de otros tipos con un breve comentario acerca del contenido de cada página o documento recogido. Son útiles para localizar información sobre un tema cuando no se conocen los principales webs sobre esa materia. A menudo están acompañados de bases de datos que facilitan la información de estos índices sustituyendo la recuperación jerárquica 
por la búsqueda directa de los términos deseados a través de un motor o buscador. Véanse por ejemplo:

- Yahoo: http://www.yahoo.es

- Galaxy: http://galaxy.einet.net

- Olé: http://www.ole.es

\subsubsection{2. Índices geográficos}

En muchas ocasiones es necesario localizar la información partiendo de la zona geográfica a la que hace referencia. Para ello se utilizan las guías o índices geográficos, en los cuáles los documentos se organizan a partir del área de la que tratan o desde la que se ha generado la página. Suelen partir de mapas sensitivos genéricos, en los que las zonas se van delimitando a medida que se eligen opciones: continentes, países, regiones, etc. Como en otras fuentes de información los índices geográficos suelen aparecer junto a buscadores desde los que se pueden localizar los documentos por términos. Véanse por ejemplo:

- Virtual Tourist: http://www.vtourist.com

- Dónde: http://donde.uji.es

- Map server for the República Argentina: http://dns.uncor.edu:8001

\subsubsection{Recopilaciones}

Selecciones de recursos realizadas por organismos, empresas o personas. En realidad son repertorios de documentos agrupados en razón de su temática o de su tipología. De esta forma tendrían aquí cabida los directorios temáticos que no emplean índices jerárquicos, los directorios de personas o instituciones, las agrupaciones de recursos de audio, vídeo, etc., en definitiva, cualquier recopilación de documentos basada en listados sin excesiva estructuración. Los criterios de calidad dependerán de los responsables de la selección, pero suelen ser un buen lugar para localizar páginas de interés como un medio de encontrar documentos seleccionados por usuarios con las mismas necesidades informativas que quien está realizando la búsqueda. Véanse por ejemplo:

- Europa on line: http://www.euroinfo.cce.es/online.html

- Listas en RedIRIS: http://www.rediris.es/list/tema/tematic.es.html

- Web médica de Rafa Bravo:

http://usuarios.bitmailer.com/rafabravo/index.htm

\subsubsection{Anillos temáticos}

El sistema de grupos o anillos temáticos se basa en la selección de un documento sobre un tema determinado y su vinculación con otro que trata del mismo asunto. Este último documento se enlazaría con un tercero de contenido similar y 
así sucesivamente. Todas las páginas seleccionadas se reúnen en un anillo temático. Este método implica que todos los documentos localizados serán específicos de la materia buscada, aunque presentan el inconveniente de que el proceso de inscripción en un grupo es manual y voluntario, lo que provoca ausencias significativas. En la actualidad hay anillos web de miles de temas, siendo por ello un buen sistema para localizar información sobre temas concretos. Véanse por ejemplo:

- Webring: http://www.webring.org

- Ringsurf: http://www.ringsurf.com

- Looplink: http://www.looplink.com

\subsubsection{Portales}

El auge comercial de los servicios de información ha dado lugar a la creación de los portales o medios para acceder desde una única vía a distintos servicios informativos de interés para el usuario. Los portales están ligados a los servicios comerciales de información, que en la actualidad suelen consistir en noticias, compra en línea, información meteorológica y cultural, guías temáticas, etc. Son fuentes de información telemática intelectuales, ya que los servicios están realizados y enlazados por personas, pero no se puede considerar que exista en ellos ningún tipo de análisis de la información, ya que suelen ser únicamente enlaces de presentación vistosa, que envían a otros servicios de información, ya sean propios o externos. Véanse por ejemplo:

- Portal Mix: http://www.portalmix.com

- Lycos: http://www.es.lycos.de

- Telepolis: http://www.telepolis.es

\subsubsection{Sitios web}

Muchos de los sitios web y páginas web individuales aportan información de interés por sí mismas, sin necesidad de enlazar con otras. En muchas ocasiones la información requerida se encuentra en una página web o un conjunto de páginas reunidas en un sitio específico, por lo que habrá que valorar la utilidad de esta fuente de información telemática como un servicio más de información en línea. Véanse por ejemplo:

- La Moncloa: http://www.la-moncloa.es

- Extra!-net: Revista de infonomía: http://www.extra-net.net/

- Las recetas de Marita: http://personal.redestb.es/amballesta/recetas.htm 


\subsection{Servicios de información con tratamiento automático}

Un buen número de sistemas de acceso a la información telemática están diseñados para facilitar de manera automática los datos requeridos por los usuarios. Por lo general se trata de bases de datos de documentos web o de otros tipos que son alimentadas por programas diseñados para tal función. Posteriormente, los programas de introducción de datos se completan con otros para recuperar dichas informaciones. Estos servicios de información son muy empleados ya que se trata de sistemas potentes en cuanto a su recuperación, además de muy eficaces, ya que suelen contener un porcentaje muy significativo de la información que está disponible en Internet.

Durante los últimos años, los buscadores o motores de búsqueda, principales servicios automáticos de información, han sido considerados la panacea en la localización de los documentos, pero los problemas que presentan en cuanto a la calidad de la información que ofrece y el excesivo ruido documental que presentan han motivado que se busquen nuevos sistemas automáticos, completados con sistemas manuales.

La diferencia esencial entre los distintos procedimientos automáticos dependerá del método de extracción y asignación de descriptores antes de introducir un documento en la base de datos correspondiente. En muchos casos no hay modo de saber cómo funcionan estos sistemas, ya que no se ofrece información de los mismos. Aunque en otras ocasiones se emplean métodos híbridos, lo normal es que los programas diseñados para la introducción de la información en estas bases de datos visiten los documentos y, bien indicen parte del mismo y extraigan descriptores del código fuente del documento, o bien recorran el texto completo o una parte significativa introduciendo la información extraída en la base de datos (16).

\subsubsection{Tratamiento automático con análisis documental}

Los programas empleados por los servicios automáticos de información funcionan de forma muy diferente. Muchos de ellos localizan la información de forma automática y la tratan de la misma manera, pero atendiendo a procedimientos basados en la asignación de descriptores bien por la frecuencia de las palabras significativas, bien por la extracción de términos del código fuente del documento (etiquetas de título, descriptores, etc.), o bien porque se emplee un tesauro que se contrasta de forma automática con la información del documento que se está analizando.

Existen buscadores o motores de búsqueda con indización cuyo programa de análisis de la información está diseñado para la extracción de los términos más significativos de un documento o bien para la asignación automática de un núme- 
ro de descriptores obtenidos mediante la comparación de los datos del documentos con el tesauro del sistema que está realizando el análisis automático. El resultado es una serie de descriptores a los que se asocia el enlace a un documento, todo lo cual es incorporado a una base de datos a la que se accede mediante un formulario de búsqueda.

Pero, no puede decirse que estos sistemas realicen un análisis documental propiamente dicho, ya que las técnicas empleadas responden a criterios de lo que los informáticos denominan indexación, algo que a menudo corresponde con la indización documental, pero que todavía, en el caso de los sistemas de recuperación de Internet, se limita a procedimientos de extracción y asignación automática de descriptores. Por este motivo este apartado de sistemas automáticos con análisis documental se ha dejado vacío.

\subsubsection{Tratamiento automático sin análisis documental}

Un gran grupo de sistemas automáticos toman toda la información de un documento o un parte significativa del mismo para introducirla en la base de datos que servirá para su recuperación posterior. En estos métodos de análisis a texto completo no puede considerarse que exista ningún tipo de análisis documental, ni siquiera automático, ya que la información se trata en bruto. Tampoco se puede considerar que exista análisis documental en aquellos sistemas que indizan/indexan automáticamente la información, ya que los procedimientos empleados difieren de las técnicas del análisis documental.

Aunque existen muchas variantes de estas fuentes de información telemática $\mathrm{y}$, a pesar de que todas pueden ser incluidas en el mismo grupo, pueden establecerse los siguientes servicios de información con tratamiento automático de la información:

\subsubsection{Buscadores}

Se trata de bases de datos de documentos web, noticias de correo, software, etc., formadas con la información sin tratar extraída de las distintas páginas web y otros documentos similares localizados por los métodos de rastreo mediante los cuáles estos sistemas recopilan los datos que identificarán a los documentos. Los programas empleados por estos buscadores utilizan la información encontrada en toda la página o en una parte significativa de la misma. Además, muchos extraen los descriptores existentes en las etiquetas meta del código fuente del documento: título, autor, materias, etc (17). La calidad de estos buscadores o motores de búsqueda dependerá sobre todo del número de documentos contenidos en su base de datos, de las posibilidades de interrogación que permitan y de la calidad de las referencias obtenidas. Véanse por ejemplo:

- Altavista: http://www.altavista.magallanes.net 
- Northern light: http://www.northernlight.com

- Webcrawler: http://www.webcrawler.com

\subsubsection{Directorios de buscadores}

Con este nombre, entre otros, se conoce a los sistemas que facilitan la consulta a distintos motores de búsqueda sin tener que conectar individualmente con cada uno de ellos, ya que desde la página del directorio o multibuscador se pueden realizar consultas de forma independiente. Su principal ventaja es que simplifican los formularios de búsquedas y que dan acceso a buscadores, índices, directorios, etc., tanto genéricos como específicos. Son las fuentes de información telemática terciaria por excelencia. Véanse por ejemplo:

- Buscopio: http://www.buscopio.com

- All in one: http://www.allonesearch.com

- AccuFind: http://www.netlocator.com

\subsubsection{Metabuscadores}

Son buscadores que no disponen de base de datos propia, sino que utilizan las de distintos motores de búsqueda simultáneamente. Cuando se realiza una consulta en un metabuscador los resultados se ofrecen indicando en qué buscador se obtuvo el documento. Unifican en un único modelo de formulario las consultas, en vez de tener que efectuarla varias veces. Por lo general, ofrecen las mismas posibilidades de interrogación que los buscadores independientes. También son llamados multibuscadores o supermotores. Véanse por ejemplo:

- Savvy search: http://www.savvysearch.com

- Cyber 411: http://www.cyber411.com

- Metacrawler: http://www.go2net.com

\section{Sistemas de acceso a la información telemática}

Como se ha comprobado en el apartado anterior, las fuentes de información disponibles en Internet son múltiples, debido tanto al tipo de documentos que incluyen como a la forma de organizar sus bases de datos o repertorios de documentos web. Ante tanta oferta el proceso de búsqueda se complica.

Por este motivo es necesario delimitar claramente determinados aspectos antes de iniciar la búsqueda de la información deseada; para ello es conveniente establecer qué se busca, para posteriormente decidir dónde y cómo buscar dicha información. 


\section{1. ¿Qué buscar?}

Cuanto más se precise en el tipo de información que se requiere más rápidamente se podrá conseguir la misma. Por este motivo es conveniente establecer si la información que se necesita estará en formato web, será un programa, una dirección de correo electrónico, etc. De la misma manera, se establecerá si se deben consultar recursos como las listas de correo o los grupos de noticias. Estableciendo los límites tipológicos de la información demandada se podrán descartar determinados sistemas de información o, por el contrario, ampliar el campo de acción de la consulta.

Por ejemplo, si deseamos información sobre la obra de un determinado músico podemos seleccionar páginas biográficas, referencias bibliográficas, archivos de audio, partituras, etc., o bien todo ello. Este es el primer tipo de especificación que conviene realizar antes de iniciar una búsqueda.

Otra delimitación en cuanto al tipo de información que se va a solicitar corresponde al nivel intelectual. No se realiza de igual forma una búsqueda de un tema genérico, que de uno específico. Cuando se quiera obtener información general es preferible hacerlo a partir de los índices y guías temáticas, ya que bastará con localizar la categoría más aproximada al tema requerido y examinar la relación de páginas incluidas. Por el contrario, cuando la consulta es especializada o concreta, es preferible utilizar otros sistemas, como los buscadores, donde la consulta se realiza directamente por los descriptores sobre los que se quiere obtener información.

Una consulta sobre páginas de banca, por ejemplo, es preferible realizarla examinando los índices y guías temáticas; mientras que si lo que es pretende es localizar el web del Banco de España, lo mejor será efectuar la consulta en un buscador.

\section{2. ¿Dónde buscar?}

Si se ha establecido el tipo de información que se necesita y el nivel científico o alcance de la misma será sencillo escoger el sistema de búsqueda más idóneo para dicha consulta. Lógicamente, si lo que se busca es la dirección de una empresa, lo mejor será acudir a un servicio de páginas amarillas. Si se pretende conocer las páginas web que traten de una zona determinada, será conveniente partir de índices geográficos, etc. Tal y como se ha dicho más arriba los temas genéricos se buscaran en índices o guías temáticas y los específicos en los buscadores.

El tipo de información indicará el sistema de información que debe ser utilizado para localizarla. Siguiendo con esta lógica, si se quieren obtener datos de 
una determinada película española, ¿dónde conseguir mejor información que en una base de datos de filmografía española?

Una vez más el número de sistemas de información es tan elevado que resulta difícil decidirse por uno u otro. Para escoger entre las fuentes de información existentes se pueden seguir estos criterios:

- Sencillez en el manejo: Que la consulta se realice de manera intuitiva, para lo que el sistema debe disponer de formularios o casillas fáciles de reconocer. Además es conveniente que se permita la consulta en el idioma del usuario.

- Rapidez en el proceso de consulta: Dependiendo del volumen de la base de datos y de los dispositivos tanto de software como de hardware la resolución de la consulta tardará más o menos tiempo. Se optará por los que habitualmente sean más rápidos.

- Base de datos de gran tamaño: Cuantas más páginas web y otros documentos se contengan mayor información se podrá obtener.

- Actualización y mantenimiento de la base: Los servicios de información deben actualizar constantemente sus contenidos, eliminar las páginas que hayan desaparecido y cambiar los enlaces cuya página de referencia ha cambiado. Se escogerán los sistemas que demuestren estar actualizados.

- Porcentaje de resultados: Se deben comparar los distintos sistemas de búsquedas en cuanto al número de registros pertinentes que ofrecen. No es tan importante el número de páginas que se ofrecen al realizar una consulta como la tasa de acierto de los resultados mostrados en primer lugar.

- Uso de operadores: Es muy importante que se permita el uso de operadores lógicos y de cualquier otro tipo para realizar una búsqueda, ya que estos sistemas permiten obtener información más relevante.

- Formatos de visualización: Es conveniente que el sistema posibilite elegir cómo se verá la información encontrada (resumida, completa, etc.) y qué número de documentos se visualizarán en una misma página (de diez en diez, etc.).

- Ayudas e información complementaria: Es importante en caso de búsquedas complejas que se informe de cómo se deben realizar las mismas. Es conveniente además, que se den datos del sistema: funcionamiento, organización, etc.

\section{3. ¿Cómo buscar?}

El acceso a los documentos se realizará por temas (browsing) cuando se pretenda obtener páginas o informaciones sobre una disciplina, mientras que la bús- 
queda se efectuará a partir de términos de consulta (searching) si se quieren datos o informaciones más concretas (18).

La búsqueda temática no suele plantear problemas, ya que se partirá de categorías genéricas hasta llegar al nivel de especificidad deseado. Una vez allí se obtendrá el listado de enlaces hacia las páginas que traten del tema buscado. Las búsquedas en las guías temáticas e índices se realizan de la materia más amplia a la más concreta.

Mayor atención requiere la búsqueda por términos, descriptores o palabras clave. Los buscadores y la mayoría de los índices disponen de un motor de búsqueda que admite varios procedimientos para su consulta:

- Búsqueda simple: En ella los términos se procesan automáticamente según los parámetros del sistema instalado en ese servidor. Ésta es aconsejable para términos simples (no compuestos) o para cuando se quieran obtener informaciones sobre varios temas de forma indistinta.

- Búsqueda avanzada: El usuario escoge la relación entre los distintos términos, pudiendo además limitar la consulta, combinarla o determinar instrucciones para obtener resultados más precisos.

Las búsquedas avanzadas son necesarias para conseguir información con un alto porcentaje de pertinencia. La mayor parte de los sistemas de búsqueda ofrecen la posibilidad de realizar este tipo de consultas. El principal problema que surge en relación a las búsquedas avanzadas es que no todos los sistemas de información actúan de la misma manera, ya que no hay coincidencia en la forma de procesar una consulta ni en el modo de establecer las opciones avanzadas. Ello obliga a que sea necesario conocer el funcionamiento de cada uno de los sistemas si que quiere obtener mayor rendimiento de los mismos.

A pesar de que no todos los sistemas de información ofrecen las mismas prestaciones, por lo general, las búsquedas avanzadas permiten operadores y delimitadores de los siguientes tipos: lógicos; posicionales; de exactitud, de truncamiento y de campo.

\subsubsection{Operadores lógicos o booleanos}

Son aquellos que posibilitan realizar una búsqueda introduciendo varios términos e indicando las relaciones de inclusión o exclusión entre los mismos. En los distintos sistemas de información telemática existentes en Internet es posible encontrar los operadores: AND, OR y NOT (19). En la página siguiente se explica la utilidad de cada uno de ellos en la tabla III.

La manera de realizar el operador puede cambiar, dependiendo del idioma del buscador y del sistema en concreto. Es frecuente que se utilicen signos para sustituir a los operadores booleanos (AND, +; NOT, -).

Scire. 4 : 2 (jul.-dic. 1998) 79-103. 


\begin{tabular}{|l|l|l|l|}
\hline Operador & Efecto & Ejemplo & Resultado \\
\hline AND & $\begin{array}{l}\text { Intersección de } \\
\text { dos términos } \\
\text { sibliotecas AND } \\
\text { salamanca }\end{array}$ & $\begin{array}{l}\text { documentos en los que } \\
\text { aparezca información sobre } \\
\text { bibliotecas y sobre Salamanca } \\
\text { de forma simultánea . Los } \\
\text { términos se buscan juntos }\end{array}$ \\
\hline OR & $\begin{array}{l}\text { Unión de dos } \\
\text { términos }\end{array}$ & $\begin{array}{l}\text { bibliotecas OR } \\
\text { salamanca }\end{array}$ & $\begin{array}{l}\text { documentos en los que } \\
\text { aparezca información sobre } \\
\text { bibliotecas o sobre Salamanca } \\
\text { de forma independiente o } \\
\text { simultánea. Se buscarán los } \\
\text { términos juntos O separados. }\end{array}$ \\
\hline NOT & $\begin{array}{l}\text { Eliminación } \\
\text { del segundo } \\
\text { término }\end{array}$ & $\begin{array}{l}\text { bibliotecas NOT } \\
\text { Salamanca }\end{array}$ & $\begin{array}{l}\text { documentos sobre bibliotecas } \\
\text { salamanca en los que no } \\
\text { aparece. El término que se } \\
\text { niega es extraído de los } \\
\text { resultados. }\end{array}$ \\
\hline
\end{tabular}

Tabla III. Operadores lógicos

\subsubsection{Operadores posicionales o de proximidad}

Otros de los métodos de búsqueda consiste en localizar términos próximos a otros términos. Supuestamente la cercanía entre términos implicará que los documentos que cumplan la ecuación de búsqueda tratarán de forma significativa de

\begin{tabular}{|l|l|l|l|}
\hline Operador & Efecto & Ejemplo & Resultado \\
\hline ADJ & $\begin{array}{l}\text { Adyacencia; } \\
\text { los términos } \\
\text { deben aparecer } \\
\text { juntos }\end{array}$ & $\begin{array}{l}\text { bibliotecas ADJ } \\
\text { universitarias }\end{array}$ & $\begin{array}{l}\text { documentos en los que } \\
\text { aparezca información sobre } \\
\text { bibliotecas y sobre Salamanca } \\
\text { de forma simultánea . Los } \\
\text { términos se buscan juntos }\end{array}$ \\
\hline NEAR & $\begin{array}{l}\text { Cercanía; los } \\
\text { términos deben } \\
\text { aparecer } \\
\text { próximos }\end{array}$ & $\begin{array}{l}\text { bibliotecas OR } \\
\text { universitarias }\end{array}$ & $\begin{array}{l}\text { documentos donde se recoja } \\
\text { información sobre bibliotecas } \\
\text { y en los que aparezca } \\
\text { próxima la palabra } \\
\text { "universitarias" en cualquier } \\
\text { contexto }\end{array}$ \\
\hline
\end{tabular}

Tabla III. Principales operadores posicionales 
los descriptores empleados en la recuperación. Los más comunes son las que se muestran en la tabla III a continuación.

Otros operadores de este tipo son FAR: un término alejado de otro; BEFORE: un término antes de otro o FOLLOWED: un término seguido de otro.

El uso de estos operadores no está demasiado desarrollado. La mayoría de los buscadores que los utilizan simplifican las órdenes ofreciendo un menú desplegable en el que se pueden elegir las opciones de cerca, lejos, junto, etc. En este apartado destaca Lycos, que posibilita el uso de varios operadores de proximidad y escoger el orden en el que deben aparecer los documentos, además del número de palabras máximas y mínimas que separen los términos buscados.

\subsubsection{Operadores de exactitud}

Se denominan así los procedimientos empleados por los distintos sistemas de búsqueda para que localicen determinados términos tal y como se han introducido en el formulario de búsqueda.

La forma más extendida es poniendo entre comillas los términos que se quieren encontrar, aunque existen otros procedimientos. Uno de ellos es elegir la opción "buscar como frase" o un equivalente en el formulario de búsqueda.

Este operador es muy útil sobre todo para cuando se quiere buscar nombres de personas, instituciones, títulos, etc., es decir, para cuando se parte de datos exactos.

Como en el resto de los operadores, en muchos buscadores esta opción puede simplificarse mediante formularios en los que se destaca la opción de búsqueda o correspondencia exacta.

\subsubsection{Operadores de truncamiento}

En ocasiones es más rápido buscar por la raíz de un término que por los distintos derivados del mismo. Para ello se emplea el truncamiento, es decir, se realiza la consulta no como un término concreto, sino como una cadena de caracteres.

Existen distintas formas de indicar el truncamiento. Por lo general, se efectúa mediante un asterisco, aunque en algunos buscadores se utiliza el símbolo del dólar o de la interrogación, por ejemplo:

bibliotec*

En el ejemplo anterior aparecerán documentos con cualquiera de los términos biblioteca, bibliotecas, bibliotecario, biblioteconomía, etc.

En este tipo de delimitadores también se incluyen los comodines o signos que sustituyen un determinado carácter en una búsqueda. Son útiles sobre todo 
cuando no se sabe cómo se escribe un término o cómo se habrá introducido en la base de datos. Por ejemplo, en

bibliotecari?s

nos aseguramos de que el sistema recuperará tanto la información relativa al descriptor "bibliotecarios" como la referente al término "bibliotecarias".

\subsubsection{Operadores de campo}

Muchos sistemas de búsqueda poseen órdenes específicas para realizar consultas en sus bases de datos. Se trata de instrucciones para localizar informaciones en el título de una página web, en su URL, en el nombre del servidor, etc. Estos delimitadores son totalmente distintos dependiendo de los buscadores que los emplean.

Altavista, por ejemplo, posibilita el uso de, entre otros, los siguientes:

- domain: busca las páginas con la palabra o frase especificada en el nombre de dominio

- image: busca las páginas que contienen alguna imagen con el nombre indicado

- link: busca las páginas que contengan algún enlace a una URL concreta

- title: busca las páginas que contienen la palabra o frase especificada en el título

- url: busca las páginas que contienen la palabra o frase especificada en la URL

Yahoo ofrece sólo la posibilidad de buscar en el título o en la URL de los documentos; para ello usa los comandos t: y u:, respectivamente.

- t: busca los términos solicitados en los títulos de las páginas web

- u: busca en la URL de los documentos

Infoseek también permite búsquedas de campo para localizar páginas que enlacen con otras páginas, para buscar información en el título del documento, en su URL o en un servidor concreto.

- link: busca documentos que enlacen con una dirección indicada

- site: busca en todas las páginas web de un servidor o sitio web solicitado

- url: busca documentos que tengan en la URL el término pedido

- title: busca los términos en los títulos de los documentos

Pero el buscador que dispone de más operadores de campo es Hot Bot, el cual posee una veintena de instrucciones para localizar dominios, tipos de archi- 
vo, mensajes de grupos de noticias, títulos de páginas, etc. Dado su interés se reproducen los mismosa continuación en la tabla IV.

\subsubsection{Otros aspectos}

Los sistemas de búsqueda permiten, por lo general, que los distintos operadores se puedan agrupar en conjuntos dependiendo de las instrucciones que se quieran ejecutar. Lo habitual es emplear los paréntesis para esta función.

\begin{tabular}{|l|l|}
\hline Operador & Acción \\
\hline domain & Busca las página de un dominio o sitio web \\
\hline depth & limita el número de resultados de un web \\
\hline feature:acrobat & Busca archivos Acrobat \\
\hline feature:applet: & Busca applet de Java \\
\hline feature:activex & Busca controles ActiveX \\
\hline feature:audio & Busca archivos de audio \\
\hline feature:embed & Busca plugins \\
\hline feature:flash & Busca el plugin Flash en HTML \\
\hline feature:form & Busca formularios en HTML \\
\hline feature:trame & Busca marcos en HTML \\
\hline feature:image & Busca archivos de imagen \\
\hline feature:script & Busca scripts \\
\hline feature:shockwave & Busca archivos Shockwave \\
\hline feature:table & Busca tablas en HTML \\
\hline feature:video & Busca archivos de video \\
\hline feature:vrml & Busca archivos VRML \\
\hline outgoinurlext & Busca archivos con una extensión concreta \\
\hline newsgroup & Busca en un grupo de noticias \\
\hline scriptlanguage & Busca páginas con Javascript o VBScript \\
\hline title & Busca en el titulo de las páginas \\
\hline after & $\begin{array}{l}\text { Busca los documentos creados o modificados tras la } \\
\text { fecha señalada }\end{array}$ \\
\hline before & $\begin{array}{l}\text { Busca los documentos creados o modificados antes de } \\
\text { la fecha señalada }\end{array}$ \\
\hline within & $\begin{array}{l}\text { Busca los documentos creados o modificados en el } \\
\text { periodo señalado }\end{array}$ \\
\hline & \\
\hline
\end{tabular}


"denominacion de origen" AND vino NOT (rioja OR navarra)

En el ejemplo se obtendrán documentos sobre denominaciones de origen de vino, excluyendo el vino de La Rioja y el de Navarra.

Es importante saber el papel que juegan las mayúsculas y los signos de puntuación en los distintos sistemas de búsqueda, ya que, por lo general, cuando se utilizan mayúsculas, acentos o signos diacríticos y de puntuación, el buscador interpreta la consulta como una búsqueda exacta. Por ello, es conveniente interrogar en minúsculas y sin acentos ni signos.

\section{Conclusión}

Internet ofrece distintos procedimientos para recuperar información como se ha visto, por lo que es conveniente conocerlos para obtener el máximo provecho de los mismos en el menor tiempo posible, algo a lo que cualquier profesional de la información debería aspirar.

En los párrafos precedentes se ha intentado mostrar cada uno de sistemas de los que un usuario dispone para acceder a la información telemática, organizándolos a partir del tratamiento y el análisis que los documentos reciben. En definitiva, se ha pretendido ofrecer un panorama de los sistemas de acceso a la información en línea y de todos los aspectos que hay que tener en cuenta para una recuperación pertinente, los cuáles pueden resumirse en los siguientes:

- Conocer los distintos métodos de acceso a la información

- Determinar la utilidad que cada uno de ellos tiene

- Establecer el valor documental de las distintas fuentes informativas

- Comparar la eficacia de sistemas iguales

- Desarrollar los sistemas avanzados de búsqueda

\section{Notas}

(1) La obra de Abadal (1997) es una buena fuente de consulta para delimitar los distintos servicios de información electrónica.

(2) Una aproximación a la variada tipología de archivos que pueden ser encontrados en Internet puede consultarse en Lardy (1997, p. 110-111).

(3) Hytelnet sigue siendo de especial utilidad para la localización y manejo de catálogos en línea que no están accesibles a través de web. La dirección principal es http://www.lights.com/hytelnet/ existiendo una copia en http://www.ucm.es /INET/hytelnet_html/start.html.

(4) Algunos lugares para esto son File Pile http://filepile.com/nc/start, Shareware.com http://www.shareware.com o Tucows, que tiene varios servidores en España: http://tucows.uam.es, http://tucows.arrakis.es, http://tucows.interplanet.es y http://www.idecnet.com/tucows.

Scire. 4 : 2 (jul.-dic. 1998) 79-103. 
(5) Los mejores lugares que todavía están accesibles vía gopher pueden consultarse en Gopher Jewels http://doradus.einet.net/GJ/ entre otras direcciones.

(6) Entre los sistemas de información especializados en correos electrónicos se encuentran Who Where? (http://www.whowhere.lycos.com) y Yahoo! People Search (http://people.yahoo.com o Listin Internet http://www.listin.com/).

(7) Un ejemplo son las páginas amarillas de la empresa Telefónica (http://www.paginasamarillas.es).

(8) La dirección 'http://amarillas.com/blancas/index.htm' es un ejemplo típico de páginas blancas.

(9) Los grupos de noticias y los mensajes enviados a los mismos pueden ser consultados en buscadores como DejaNews (http://www.dejanews.com/).

(10) La mayor parte de las listas de distribución españolas están en el servidor de RedIRIS. La relación de las mismas se encuentra en 'http://www.rediris.es/list/listnac.es.html'. Un completo directorio de listas de distribución es CataList (http://segate.sunet.se/lists/listref-eng.html).

(11) Cada vez son más frecuentes los buscadores especializados en imágenes, como AVPhotoFinder (http://image.altavista.com). En el apartado de mapas destaca Mapquest (http://www.mapquest.com).

(12) El servidor de Real Audio (http://www.realaudio.com/) es un buen lugar para encontrar archivos musicales.

(13) Existen múltiples directorios desde los que localizar los canales de conversación en Internet. Uno de ellos es Liszt (http://www.liszt.com/chat/).

(14) Un completo directorio de webcams es El ojo (http://dominios.net/elojo/index.htm).

(15) El breve estudio de Cleveland (1998) para la IFLA-UDT es muy útil para delimitar los concepto relacionados con las bibliotecas digitales.

(16) Un buen sitio web desde el que informarse de cómo funcionan los buscadores es Search Engines Watch (http://searchenginewatch.com).

(17) Codina (1997a, 1997b) explica claramente el doble proceso en el que se basa el funcionamiento de los motores de búsqueda.

(18) Dentro de la bibliografía existente sobre el browsing y el searching en Internet es interesante el artículo de Chen (1998).

(19) Otro operador que se emplea es XOR, que realiza una búsqueda combinando diversos términos, pero indicando la incompatibilidad entre alguno de ellos.

\section{Referencias}

Abadal, E. (1997). Els serveis d'informació electrònica, què son i per a què serveixen. Barcelona : Universitat, 1997.

Abadal, E. ; Perpinyà, M. (1998). Trobar informació a Internet: alguns consells pràctics. // Cercar i col.locar informació en el World Wide Web. Barcelona: Llibres de l'Index, 1998, P. 109-137.

Acevedo, F. ; et al. (1998). Buscadores de Internet. Madrid: Paraninfo, 1998. 
Ackermann, E. ; Hartman, K. (1998). Searching and researching on the Internet and the World Wide Web. Wilsonville : Franklin, Beedle \& Associates, 1998.

Andrieu, O. (1997). Cómo buscar y encontrar en Internet. Barcelona : Gestión 2000, 1997.

Bardi, Luca (1998). Prende forma la digital library : progetti, tecnologie, problemi. // Biblioteche oggi. (dic. 1998) 6-12.

Bates, Marcia J. (1998). Indexing and access for digital libraries and the internet: human, database, and domain factors. // Journal of the American Society for Information Science. 49:13 (1998) 1185-1205.

Chen, H. ; et al. (1998) Internet browsing and searching : user evaluations of category maps and concepts space techniques. // Journal of the American Society for Information Science. $49: 7$ (1998) 582-603.

Cleveland, Gary. (1998). Digital libraries : definitions, issues and challenges. Ottawa : IFLA International Office for UDT, 1998. (Occasional paper; 8).

Codina, L. (1998). Bases lògiques del funcionament dels motors de cerca d'Internet: anàlisis critica i prospectiva. // Cercar i col.locar informació en el World Wide Web. Barcelona : Llibres de 1'Index, 1998. P. 139-176.

Codina, L. (1997a). Cómo funcionan los servicios de búsqueda en Internet : un informe especial para navegantes y creadores de información (Parte I). // Information World en Español. 6 : 5 (mayo 1997) 22-27.

Codina, L. (1997b). Cómo funcionan los servicios de búsqueda en Internet : un informe especial para navegantes y creadores de información (Parte II). // Information World en Español 6 : 6 (junio 1997) 18-27.

Díaz Ferreira, M. A. (1998). Buscadores temáticos. // Iworld. 1 (en. 1998). URL: <http://www.idg.es/iworld/199801/articulos/btematicos.asp>.

Glossbrenner, A.; Glossbrenner, E. (1998). Search engines for the World Wide Web. 2nd ed. Brekeley : Peachpit, 1998.

Grau, A. (1998). Buscar por la web. // Iworld. 10 (nov. 1998). URL:<http://www.idg.es/iworld/199811/articulos/buscadores.asp>.

Lardy, J.-P. (1996). Les outils de recherche d'information sur Internet: guides, listes thématiques et index. // Documentaliste, Sciences de l'information. 33 : 1 (1996) 33-39.

Lardy, J.-P. (1997). Recherche d'information sur l'Internet : outils et méthodes. Paris : ADBS, 1997.

Le Guelvoit, A. (1998). Les outils de recherche du web: limites et aléas du référencement. // Documentaliste, Science de l'information. 35 : 6 (1998) 315-320.

Leloup, C. Motores de búsqueda e indexación. Barcelona : Gestión 2000, 1998.

Maldonado Martínez, Á. ; Fernández Sánchez, E. (1998). Evaluación de los principales "buscadores" desde el punto de vista documental: recogida, análisis y recuperación de recursos de información. // Jornadas Españolas de Documentación (6. Valencia. 1998). Los sistemas de información al servicio de la sociedad. Valencia : FESABID; AVEI, 1998. P. 529-551.

Marcos Mora, M. C. (1998). Motores de recuperación de información: un análisis comparativo (parte I). // El profesional de la información. 7 : 1-2 (en.-feb. 1998) 18-22. 
Marcos Mora, M. C. (1998). Motores de recuperación de información : un análisis comparativo (parte II). // El profesional de la información. 7 : 3 (marzo 1998) 13-20.

Merlo Vega, J. A.; Sorli Rojo, Á. (1998). Acceso a la información y suministro de documentos en la era Internet. // Jornadas Bibliotecarias de Andalucía (10. Jerez de la Frontera.1998). ¿Biblioteca real frente a biblioteca virtual?. Málaga : Asociación Andaluza de Bibliotecarios; Diputación, 1998. P. 283-299.

Parra Pérez, B. (1998). Cómo buscar información en Internet. Madrid : Anaya Multimedia, 1998.

Senso, J. A. (1998). Herramientas para realizar búsquedas en Internet: una revisión. // El profesional de la información. 7 : 1-2 (en.-feb. 1998) 24-25.

Vaquero Pulido, J. R. (1997). Recuperación de la información en Internet : motores y otros agentes de búsqueda. // Scire. 3 : 2 (jul.-dic. 1997) 85-100. 